Roberto Farina

Eriberto Bressan

Andrei Taut

Alessandro Cucchi

Leonardo Trombelli

\section{Plasma rich in growth factors in human extraction sockets: a radio- graphic and histomorphometric study on early bone deposition}

Key words: alveolar process, bone remodeling, CD68 antigen, growth factors, histology, osteocalcin, plasma, platelet-rich plasma, tooth extraction, von Willebrand factor

\author{
Roberto Farina, Leonardo Trombelli, Research \\ Centre for the Study of Periodontal and \\ Peri-Implant Diseases, University of Ferrara, \\ Ferrara, Italy \\ Eriberto Bressan, Department of Medical and \\ Surgical Specialties, University of Padova, Padova, \\ Italy \\ Andrei Taut, Department of Periodontics and Oral \\ Medicine, School of Dentistry, University of \\ Michigan, Ann Arbor, MI, USA \\ Alessandro Cucchi, Department of Clinical and \\ Experimental Medicine, University of Ferrara, \\ Ferrara, Italy \\ Corresponding author: \\ Leonardo Trombelli \\ Research Centre for the Study of Periodontal and \\ Peri-Implant Diseases \\ University of Ferrara \\ Ferrara, Italy \\ Tel.: +390532 205277 \\ Fax: +390532202329 \\ e-mail: leonardo.trombelli@unife.it
}

Authors' affiliations:

\author{
Date: \\ Accepted 30 July 2012 \\ To cite this article: \\ Farina R, Bressan E, Taut A, Cucchi A, Trombelli L. Plasma \\ rich in growth factors in human extraction sockets: \\ radiographic and histomorphometric study on early bone \\ deposition. \\ Clin. Oral Impl. Res. 24, 2013, 1360-1368 \\ doi: $10.1111 /$ clr. 12033
}

\begin{abstract}
Objectives: To determine whether and to what extent the additional application of plasma rich in growth factors (PRGF) to an extraction socket may influence the early bone deposition, as assessed by micro-computed tomography (micro-CT) scan as well as histomorphometric markers.

Material and methods: Twenty-eight patients (age range: 34-74 years) contributing 36 extraction sockets were included in the study. Sockets were either treated with PRGF (PRGF group; 18 sites in 11 patients) or left to spontaneous healing (control group; 18 sites in 17 patients). Radiographic and histomorphometric analysis was performed on bone cores trephined from each healing socket after 4-6 (T1) or 7-10 (T2) weeks of healing.

Results: Patients treated with PRGF application showed (i) similar bone volume and tissue mineral content, (ii) a trend, although not statistically significant, toward a greater number of CD68+ cells (at T1 and T2) and VVW+ cells (at T1), and (iii) a similar OCN staining score throughout the study, when compared with control group.

Conclusions: Plasma rich in growth factors-treated group did not show any enhancement in early ( 4 and 8 weeks) bone deposition compared with control group.
\end{abstract}

The processes involved in the healing of an extraction socket have been evaluated in different animal (Kuboki et al. 1988; Lin et al. 1994, 2011; Lekic et al. 2001; Cardaropoli et al. 2003, 2005; Kanayama et al. 2003; Sato \& Takeda 2007; Vignoletti et al. 2012) and human (Clafin 1936, Mangos 1941; Christopher 1942; Amler et al. 1960, 1964; Boyne 1966; Amler 1969; Evian et al. 1982; Trombelli et al. 2008) models (see Farina \& Trombelli 2012 for review). Recently, a study conducted on human extraction sockets reported that the rate of healing markedly varies between subjects, and the process of tissue remodeling, that is, the replacement of woven bone with lamellar bone and marrow, can be incomplete even at 24 weeks (Trombelli et al. 2008). Thus, while a provisional connective tissue consistently forms within the first weeks of healing, the interval during which mineralized bone is laid down is much less predictable. At present, the incompleteness of the information regarding the timing of bone deposition and maturation into the healing socket as well as the wide variability observed between individuals limit the possibility to determine the proper time for implant placement following tooth removal.

During the last decade, several studies have been conducted to determine the potential role of biological agents and graft biomaterials in modulating the healing dynamics of the extraction socket (Farina \& Trombelli 2012). As a platelet concentrate, platelet-rich plasma (PRP, i.e., preparations characterized by a high concentration of platelets, obtainable by a single- or a double-step centrifugation of autologous blood) contains a number of different growth factors, including PDGF, TGF-b, and IGF /Okuda et al. 2003; Trombelli \& Farina 2008), which may potentially exert a positive effect on cell lines involved in wound healing (Lacci \& Dardik 2010) and bone repair (Alsousou et al. 2009). Among the available PRP preparations, PRGF technology enables the transformation of platelets and endogenous fibrin potential into different formulations with varying therapeutic potential (Anitua et al. 2007a, 2010). PRGF technology has given rise to an optimized and 
safer product known as PRGF, which circumvents many of the limitations of other reported PRP and sealant products and offers new perspectives for future regenerative procedures (Anitua 1999; Chen et al. 2010). In particular, preliminary observational studies indicated that PRGF may exert beneficial effects when applied to healing sockets (Anitua 1999; Nazaroglou et al. 2009; Mozzati et al. 2010). PRGF has been claimed to induce a faster re-epithelialization and a consistently higher content of mineralized bony trabeculae at 10-16 weeks after tooth extraction in humans (Anitua 1999).

The aim of the present clinical study was to determine whether and to what extent the additional application of PRGF to an extraction socket may influence the early bone deposition, as assessed by micro-computed tomography (micro-CT) as well as histomorphometric analysis.

\section{Material and methods}

\section{Experimental design}

The present study was designed as a parallelarm, open-label, controlled clinical trial and conducted in alignment with a previously published experimental model for the evaluation of extraction socket healing in humans (Trombelli et al. 2008).

Treatments were performed by three operators at three clinical centers. A clinical operator with specific training and clinical skill as to the treatment with PRGF selected all PRGF-treated sites, whereas the other two trained clinical operators selected all control sites (i.e., extraction sites left to spontaneous healing). Each clinician performed all experimental (either PRGF or control) procedures from tooth extraction to biopsy collection. Patient allocation to PRGF or control treatment was dependent on which center he/she attended for professional dental care.

All eligible subjects received detailed information regarding the experimental protocol. The type of information was dependent on the treatment to be performed (extraction with or without PRGF placement into the socket). Each patient provided a written informed consent before participation. All the clinical procedures were performed in accordance with the Declaration of Helsinki and Good Clinical Practice Guidelines (GCPs). Procedures for specimen processing and histomorphometric analysis received a determination of "not regulated status" (approval code: HUM00054522) from the Medical School Institutional Review Board,
University of Michigan (Ann Arbor, MI, USA).

\section{Study population}

The following inclusion/exclusion criteria were checked to determine the inclusion of the subjects in the study:

1. Age. Subjects under 18 years were excluded;

2. Health status. Subjects with positive medical history for severe systemic pathologies, acute or chronic, pregnant or lactating women, subjects who assumed drugs influencing osseous metabolism (e.g., bisphosphonates, corticosteroids) were excluded from the study.

\section{Characteristics of extraction sockets}

Eligibility criteria were represented by the presence of at least one post-extraction site related to the extraction of a single-rooted tooth or single roots of hemisected mandibular molars. The following post-extraction sites were excluded:

1. Oro-sinusal communication.

2. Endodontic periapical lesions with diameter $>5 \mathrm{~mm}$ (radiographically determined).

3. Intraosseous component of the extraction socket (i.e., the distance from the most apical alveolar crest to the bottom of the socket) $<5 \mathrm{~mm}$.

\section{Extraction and post-extraction protocol}

In all cases, the extraction procedures were performed with a flapless approach. Roots of mandibular molars were separated with rotating burs and scalpels avoiding any trauma to the inter-radicular bone. All teeth/roots were gently mobilized with luxators or elevators and extracted with forceps, using rotational movements and vertical traction to minimize the trauma to the socket walls. In PRGF group, PRGF was placed to fill the intraosseous component of the extraction defect. Extraction sites were left to heal by secondary intention. If placed, sutures were removed 1 week postoperatively. Local antimicrobial therapy (CHX $0.12 \%$ mouthrinse; $10 \mathrm{ml}$ t.i.d for 7 days) was prescribed. If an infective complication required a systemic antibiotic therapy, the extraction site was excluded from the study.

\section{PRGF preparation}

Blood was obtained prior to administration of anesthesia. Twenty $\mathrm{ml}$ of autologous blood was drawn from the peripheral vein of each patient and stored using $3.8 \%$ trisodium citrate solution as anticoagulant. The tubes were centrifuged at $580 \mathrm{~g}$ for $8 \mathrm{~min}$ (PRGF System IV, BTI Biotechnology Institute, Vitoria, Spain) at room temperature. The blood was separated into three basic components: red blood cells, which appeared at the bottom of the tube, PRGF in the middle of the tube, and plasma poor in growth factors (PPGF) at the top of the tube. The fraction $(0.5 \mathrm{ml})$ located immediately above the erythrocytes was collected from each tube and transferred to sterile tubes. This procedure results in a platelet enrichment of PRGF ranging from 2.6-fold $\left(0.5 \times 10^{6}\right.$ platelets $\left./ \mu 1\right)$ to 3.6 -fold $\left(0.7 \times 10^{6}\right.$ platelets $\left./ \mu 1\right)$ over the baseline concentration in whole blood (Anitua et al. 2007b, 2011). The activation of PRGF was accomplished with calcium chloride $150 \mathrm{lL}$ at $10 \%$ added per $1-\mathrm{ml}$ fraction of PRGF) 10 15 min prior to PRGF placement in the extraction socket.

\section{Specimen harvesting procedure}

The specimen harvesting procedure has been previously described (Trombelli et al. 2008). Briefly, before tooth extraction, an alginate impression of the experimental area was taken. On the cast model, a resin/silicon stent was prepared and adapted on the teeth adjacent to the tooth to be extracted. The stent was used to determine the topographic location of the extraction site during the biopsy procedure by means of a reference groove/hole prepared in correspondence to the tooth to be extracted.

Immediately after tooth extraction, the distance from the stent to the bottom of the alveolus was measured by means of a calibrated probe. This measurement ensured that, at time of biopsy sampling, the trephine bur collected the specimen in the area of newly formed tissue, that is, coronal to the original (post-extraction) location of the bottom of the alveolus (thus avoiding to sample the pre-existing bone).

Extraction sites of test group were treated with PRGF. Control sites were left to spontaneous healing (i.e., without the additional use of PRGF). Experimental procedures in test and control site differed exclusively for the use of PRGF.

Biopsies were scheduled at 4 weeks or 8 weeks after tooth extraction. After flap elevation, the specimen was collected with a trephine bur (internal ø: $2 \mathrm{~mm}$, external ø: $3 \mathrm{~mm})$. The minimal length of the specimen was $5 \mathrm{~mm}$. The specimen was collected at the center of the socket, as determined by the stent, with the bur kept parallel to the 
long axis of the adjacent tooth (or perpendicular to the alveolar crest profile). The apical portion of the specimen, once dried, was marked with a fine indelible chine pen. If the specimen was fragmented into $(2 / 3)$ different pieces, each fragment was separately stored, and the exact apico-coronal orientation of the specimen was maintained. In all cases, the biopsy harvesting was performed concomitantly with implant positioning at the experimental site.

\section{Micro-CT analysis}

Non-decalcified extraction core specimens were scanned at a resolution of $18 \times 18$ $\times 18 \mu \mathrm{m}^{3}$ voxels using a Pxs5-928EA conebeam micro-CT system (GE Healthcare, Little Chalfont, UK). GEHC MicroView Analysis Plus ${ }^{\circledR}$ software (GE Healthcare) was used to create three-dimensional (3D) reconstructions of bone core specimens from the scans as previously described (Park et al 2007). Region of interest (RoI) was represented by the entire extraction core or fragmented tissues samples. Threshold grayscale value was determined for each specimen by a single masked and calibrated examiner (A.T.). The average threshold grayscale value was calculated and used as the representative value to assess bone volume (BV) and tissue mineral content (TMC). Tissue mineral density (TMD) was calculated for all extraction core specimens as TMC/ $\mathrm{BV}$.

\section{Histological evaluation}

Specimen storage and processing

The collected specimens were immediately fixed in $10 \%$ formalin until micro-CT analysis. Following $\mu \mathrm{CT}$ scanning, extraction cores were decalcified for 2 weeks in $10 \%$ ethylene diaminetetraacetic acid (EDTA) at $4^{\circ} \mathrm{C}$, transferred to $70 \%$ ethanol, and stored at $4^{\circ} \mathrm{C}$ until paraffin embedding. Specimens were embedded in paraffin, cut into $5 \mu \mathrm{m}$ sagittal plane sections, and mounted on slides. Sections representing the central-most portion of the bone core were chosen for histological and immunohistochemical staining purposes.

Selected sections were stained with hematoxylin and eosin, as well as Masson's trichrome. Remaining unstained slides were used to perform immunohistochemical staining to identify the presence of vasculature/ endothelial cells (von Willebrand factor vWF), osteoblasts/bone matrix synthesis (Osteocalcin - OCN), and macrophages, giant cells (CD68). In preparation for immunohistochemical staining, paraffin-embedded sec- tions were de-paraffinized in xylene $(2 \times$ $2 \mathrm{~min}$ ) and rehydrated through graded alcohols. For von Willebrand factor and CD68 antibodies, antigen retrieval was performed using a Decloaking Chamber (Biocare Medical, Walnut Creek, CA, USA) where slides were immersed in $1 \times$ Target Retrieval Solution (Dako, Glostrup, Denmark) and boiled for $2 \mathrm{~min}$ at $125^{\circ} \mathrm{C}$ and $5 \mathrm{~min}$ at $100^{\circ} \mathrm{C}$, respectively, and then allowed to cool at room temperature for approximately $45 \mathrm{~min}$ (Nor et al. 2001). Immunohistochemical staining was completed using the manufacturer instructions of the appropriate antibody detection kit. Primary antibody incubations were performed at room temperature for the recommended time. $0.1 \%$ Tris-phosphatebuffered saline (TPBS) was used as the wash buffer, and diaminobenzidine (DAB) as the substrate/chromogen for all immunohistochemical staining procedures. Sections were rinsed in phosphate-buffered saline (PBS) and distilled water, counterstained, and mounted. For each antibody tested, one section was incubated in serum and served as the negative control.

\section{Histomorphometric analysis}

The histomorphometric analysis was carried out by a single calibrated examiner (A.T.), masked as to the treatment allocation codes and observational interval.

Histological and immunohistochemical images were captured using Nikon Eclipse $50 i$ microscope (Nikon Inc., Melville, NY, USA) fitted with a Nikon Digital Sight DS U1 camera (Nikon Inc.). Quantitative histomorphometric analysis was completed using NIS Elements Basic Research imaging software (Nikon Inc.). The central-most portion of the extraction core was chosen for histomorphometric analysis, and standardized RoIs in the coronal, middle, and apical portions of the sample were analyzed (Kaigler et al. 2010). The RoI was sized to encompass functionally relevant regions of the sample and also provide a standardized viewing window at $20 \times$ magnification.

Red-green-blue (RGB) colorimetric thresholding values were identified, yielding the most representative depiction of dark brown DAB labeling within each RoI. The average RGB intensity value was calculated for vWF and CD68 immunohistochemical staining and used to individually quantify the number of cells and area coverage. In the NIS Elements Basic Research software, groups of neighboring pixels are defined as objects. The number of objects was assumed to correlate to the number of vWF- and CD68-positive cells (objects measuring $<10 \mu \mathrm{m}^{3}$ were ecluded to prevent the quantification of artifacts). The area of positive DAB staining corresponded to area occupied by vWF- and CD68positive cells.

Average pixel intensity (ranging from 0 to 255) was used to quantify the relative expression of OCN in tissue. Low pixel intensities corresponded to the color black, while the highest intensities indicating the presence of white, with the brown DAB substrate measuring approximately 150 . Thus, the OCN score was inversely correlated to average pixel intensity (a.p.i.): a.p.i $=180 \div 192$ : score 3; a.p. $\mathrm{i}=192.0001 \div 204: \quad$ score 2 ; a.p.i $=204.0001 \div 216: \quad$ score $\quad 1$; a.p.i $=216.0001$ or above: score 0 .

\section{Statistical analysis}

The patient was regarded as the statistical unit. For patients contributing multiple sites, data were averaged to give a patient value. Data were entered in a unique database and analyzed using a statistical software (STATISTICA; Statsoft Italia, Vigonza, Italy). Data were expressed as mean and standard deviation (SD) as well as median and interquartile range (IR). Comparisons were performed using $\chi^{2}$ test, Fisher's test, and Mann-Whitney $U$-test.

Bone volume was regarded as the primary outcome variable. As data on BV to evaluate the healing process of human extraction sockets could not be retrieved, a post hoc power analysis was performed. Assuming a standard deviation in BV of $2.0 \mathrm{~mm}^{3}$ and an expected inter-group difference in $\mathrm{BV}$ of $2.0 \mathrm{~mm}^{3}, 1.5 \mathrm{~mm}^{3}$, or $1.0 \mathrm{~mm}^{3}$, a sample of 10 patients (five patients/group) had a power of $89.0 \%, 56.2 \%, 35.0 \%$, respectively, in detecting a significant inter-group difference (at $P=0.05$ ).

\section{Results}

\section{Study population and protocol deviations}

Twenty-eight patients (mean age: 55.2 years, minimum-maximum values: $34-74$ years; 13 males, 15 females) contributing 36 extraction sites were included in the study.

\section{Control group}

Seventeen patients (mean age: 57.0 years, range: 34-74 years; eight males, nine females) contributed 18 extraction sites. Teeth were extracted owing to root fracture $(n=9)$, caries $(n=3)$, implant-supported prosthetic treatment planning $(n=5)$, or failure of endodontic treatment $(n=1)$. 


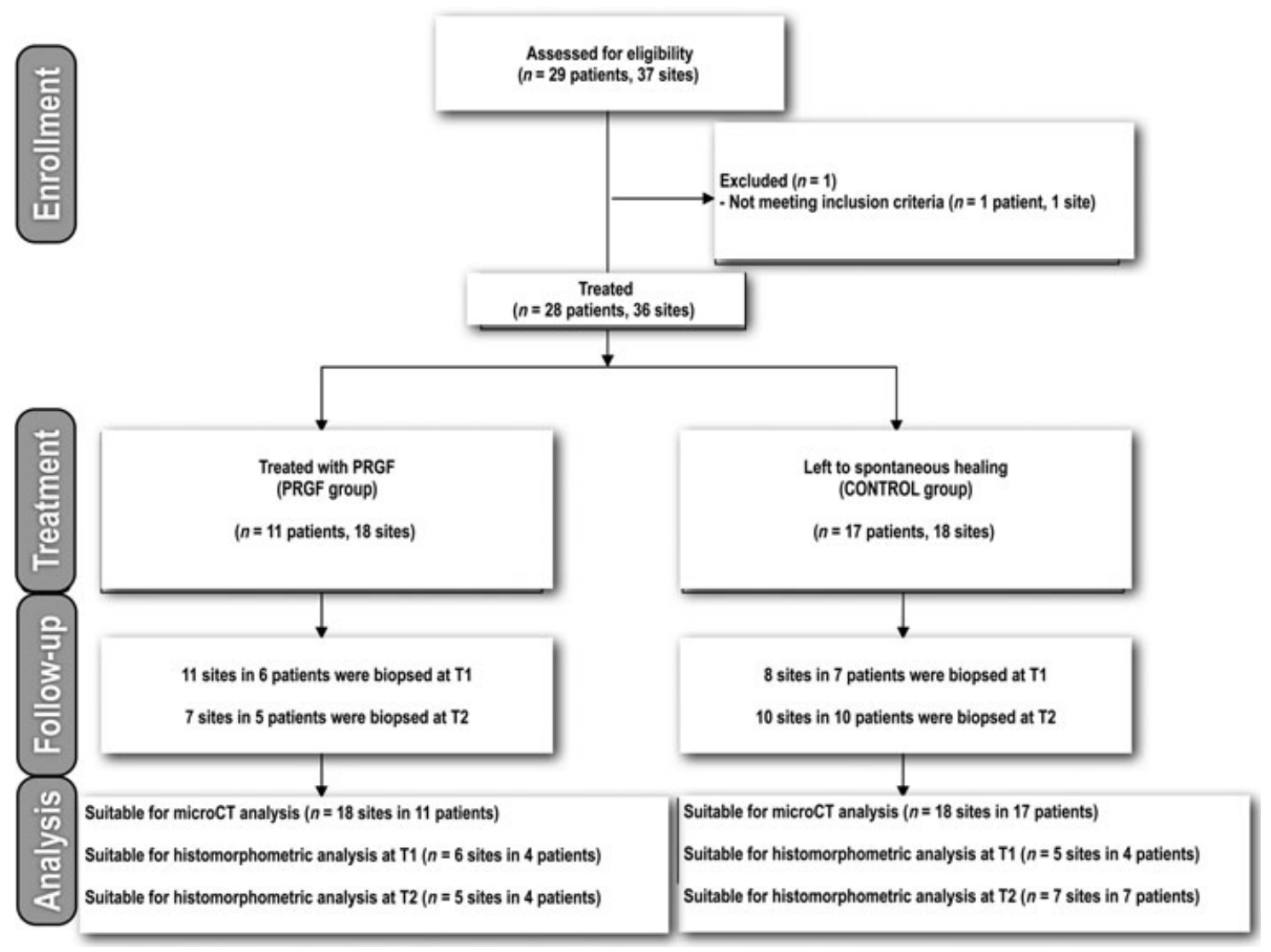

Fig. 1. Flow chart of the study.

Eight specimens in seven patients (mean age: 61.4 years, range: 51-74 years; three males, four females) were collected after 4 weeks of healing. Ten specimens in 10 patients (mean age: 53.9 years, range: $34-73$ years; five males, five females) scheduled for biopsy at 8 weeks were collected after 7 weeks (three sites) or 8 weeks (seven sites) of healing.

\section{PRGF group}

Eleven patients (mean age: 51.0 years, range: 42-64 years; five males, six females) contributed 18 extraction sites. Teeth were extracted owing to severe bone loss (destructive periodontitis) $(n=6)$, root fracture $(n=5)$, caries $(n=4)$, implant-supported prosthetic treatment planning $(n=2)$, or failure of endodontic treatment $(n=1)$.

Eleven specimens in six patients (mean age: 51.2 years, range: $48-57$ years; three males, three females) scheduled for biopsy at 4 weeks were collected after 4 weeks (nine sites in four patients), 5 weeks (one site), or 6 weeks (one site) of healing. Seven specimens in five patients (age: 54.0 years, range: 42-64 years; two males, three females) scheduled for biopsy at 8 weeks were collected after 8 weeks (four sites in three patients), 9 weeks (two sites in one patient), or 10 weeks (one site) of healing.

Table 1. Patient- and site-related characteristics in PRGF and control groups

\begin{tabular}{|c|c|c|c|c|}
\hline & & Control & PRGF & $P$ value \\
\hline \multicolumn{5}{|l|}{ Patient level } \\
\hline \multirow[t]{2}{*}{ Number of patients } & $\mathrm{T} 1$ & 7 & 6 & - \\
\hline & $\mathrm{T} 2$ & 10 & 5 & \\
\hline \multirow[t]{3}{*}{ Age (years) } & Mean & 57.0 & 52.5 & 0.175 \\
\hline & Median & 60.0 & 51.0 & \\
\hline & Range & $34-74$ & $42-64$ & \\
\hline Gender (number of females) & & 9 & 6 & 1 \\
\hline $\begin{array}{l}\text { Smoking status (smokers/former } \\
\text { smokers/never smoked) }\end{array}$ & & $0 / 5 / 12$ & $5 / 5 / 1$ & $<0.001$ \\
\hline \multicolumn{5}{|l|}{ Site level } \\
\hline \multirow{2}{*}{ Number of sites } & $\mathrm{T} 1$ & 8 & 11 & - \\
\hline & $\mathrm{T} 2$ & 10 & 7 & \\
\hline \multirow{2}{*}{$\begin{array}{l}\text { Site distribution according to tooth type } \\
\text { (incisor/canine/ } \\
\text { premolar/single root of mandibular molar) }\end{array}$} & $\mathrm{T} 1$ & $2 / 2 / 4 / 0$ & $1 / 2 / 6 / 2$ & N.D. \\
\hline & $\mathrm{T} 2$ & $4 / 0 / 5 / 1$ & $0 / 0 / 7 / 0$ & N.D. \\
\hline \multirow{2}{*}{$\begin{array}{l}\text { Site distribution according to location } \\
\text { (maxillary/mandibular) }\end{array}$} & T1 & $7 / 1$ & $5 / 6$ & 0.026 \\
\hline & $\mathrm{T} 2$ & $6 / 4$ & $5 / 2$ & 1 \\
\hline
\end{tabular}

Specimens harvested after 4-6 weeks and 7 -10 weeks were pooled for data analysis into two distinct observation intervals: $\mathrm{T} 1$ and $\mathrm{T} 2$, respectively.

The CONSORT flow chart of the study is illustrated in Fig. 1. Patient- and site-related characteristics of PRGF and control groups are reported in Table 1. Treatment groups were significantly different for smoking status $(P<0.001)$.

\section{Micro-CT analysis}

Representative micro-CT scans of the study specimens in control and PRGF groups are reported in Fig. 2.

Bone volume $\left(\mathrm{mm}^{3}\right)$, TMC (mg), and TMD $\left(\mathrm{mg} / \mathrm{cm}^{3}\right)$ in control and PRGF groups are shown in Table 2.

In the control group, newly formed bone occupied a variable part of the specimen at T1. Although a trend toward greater BV and 

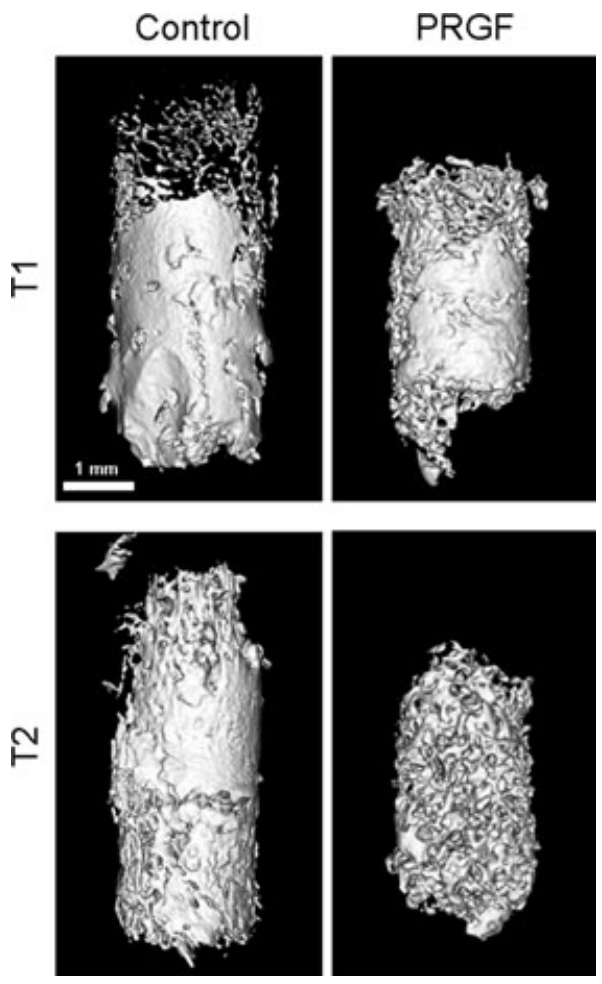

Fig. 2. Representative micro-CT scans of the study specimens in control and PRGF groups.

TMC values was evident at T2, the difference in $\mathrm{BV}$ and $\mathrm{TMC}$ from $\mathrm{T} 1$ to $\mathrm{T} 2$ did not reach the statistical significance.

In PRGF group, limited BV and TMC were observed at T1. Both BV and TMC showed a slight (not statistically significant) increase at T2. No significant differences in BV and TMC were observed between groups at either $\mathrm{T} 1$ or $\mathrm{T} 2$.

In both control and PRGF groups, a similar TMD was observed between $\mathrm{T} 1$ and $\mathrm{T} 2$. No significant differences in TMD were observed between groups at either $\mathrm{T} 1$ or $\mathrm{T} 2$ (Table 2).

\section{Histomorphometric analysis}

Five specimens in four control patients and six specimens in four PRGF patients were suitable for histomorphometric analysis at $\mathrm{T} 1$. At $\mathrm{T} 2$, histomorphometric assessment was performed on seven specimens in seven

T1
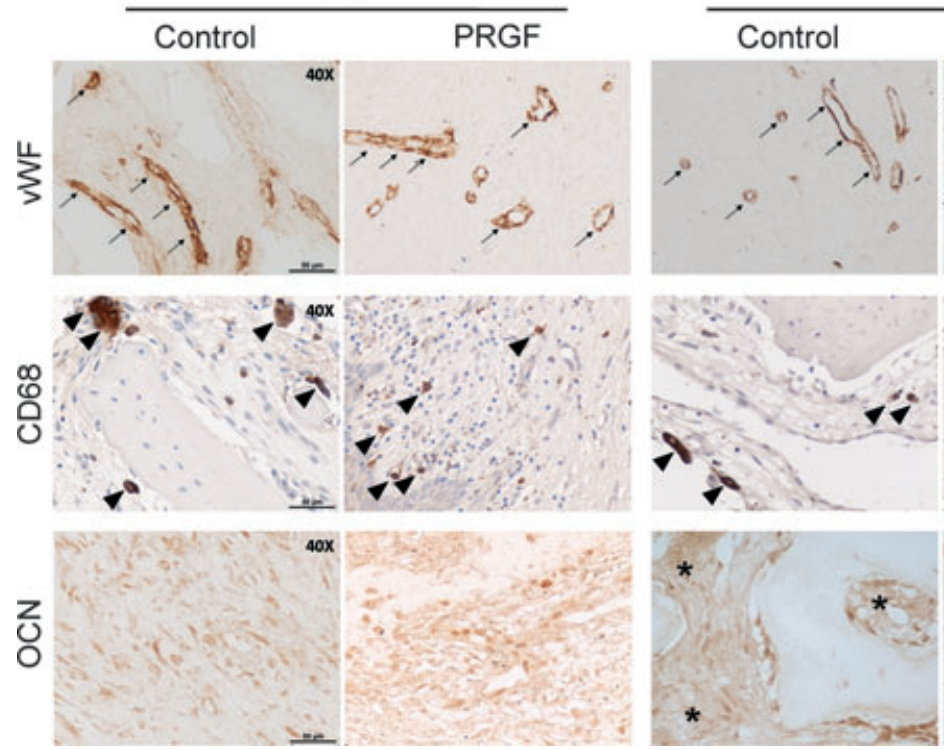

Fig. 3. Representative specimens for each immunohistochemical staining in control and PRGF groups. Black arrows depict vWF/Factor VIII-positive endothelial cells forming blood vessels. Black triangles represent CD68-positive macrophages. Asterisks depict the osteocalcin-positive early-stage and mature mineralized tissue matrix.

control patients and five specimens in four PRGF patients (Fig. 1). Specimens that exhibited significant damage or large artifacts were excluded from the histomorphometric analysis. Also omitted from the histomorphometric analysis were tissue sections not representative of the most central portion of the bone core. Representative specimens for each immunohistochemical staining in control and PRGF groups at $\mathrm{T} 1$ and $\mathrm{T} 2$ are reported in Fig. 3.

\section{CD68}

A tendency to show a higher number of CD68+ cells was observed in PRGF group at $\mathrm{T} 1$ and $\mathrm{T} 2$; however, no significant differences were found between groups at both observation intervals (Table 3). While an increase in CD68+ cells was observed from $\mathrm{T} 1$ to $\mathrm{T} 2$ in control group, a slight decrease occurred in PRGF group. However, withingroup changes in the number of CD68+ cells were not statistically significant for both groups (Table 3). von Willebrand factor

The number of vWF+ objects, which was assumed to correlate to the number of vWF+ cells, was higher, although not statistically significantly, at $\mathrm{T} 1$ in PRGF compared with control group (Table 3). In contrast, similar presence of $\mathrm{vWF}+$ cells (assessed as either number of objects or area) was observed at $\mathrm{T} 2$, owing to a decrease (although not significant) in vWF+ cells in the PRGF group (Table 3).

\section{Osteocalcin}

Osteocalcin score showed a statistically significant decrease from $\mathrm{T} 1$ to $\mathrm{T} 2$ for both control and PRGF group $(P=0.006$ and $P=0.029$, respectively). No significant differences in OCN score were observed between groups at both observation intervals (Table 3).

\section{Discussion}

The present study was performed to evaluate the early phases of bone deposition in human

Table 2. Results of micro-CT analysis: bone volume (BV, $\mathrm{mm}^{3}$ ), tissue mineral content (TMC, $\left.\mathrm{mg}\right)$, and tissue mineral density (TMD, $\mathrm{mg} / \mathrm{cm}^{3}$ ). Data are expressed as mean and standard deviation (SD) as well as median and interquartile range (IR)

\begin{tabular}{|c|c|c|c|c|c|c|c|c|c|c|}
\hline & & \multicolumn{3}{|c|}{ Bone volume $\left(\mathrm{mm}^{3}\right)$} & \multicolumn{3}{|c|}{ Tissue mineral content $(\mathrm{mg})$} & \multicolumn{3}{|c|}{ Tissue mineral density $\left(\mathrm{mg} / \mathrm{cm}^{3}\right)$} \\
\hline & & Control & PRGF & $P$ value & Control & PRGF & $P$ value & Control & PRGF & $P$ value \\
\hline \multirow[t]{2}{*}{ T1 } & Mean (SD) & $3.1(3.4)$ & $1.4(2.0)$ & 0.177 & $1.8(2.0)$ & $0.7(1.0)$ & 0.177 & $519.4(123.5)$ & $480.0(97.6)$ & 0.329 \\
\hline & Median (IR) & $2.5(0.6-4.6)$ & $0.3(0-2.6)$ & & $1.5(0.3-2.6)$ & $0.2(0-1.3)$ & & $567.5(466.6-603.2)$ & $460.8(445.9-477.2)$ & \\
\hline \multirow[t]{2}{*}{$\mathrm{T} 2$} & Mean (SD) & $4.5(2.7)$ & $3.2(2.9)$ & 0.792 & $2.5(1.7)$ & $1.7(1.8)$ & 0.662 & $538.4(106.6)$ & $485.8(73.1)$ & 0.429 \\
\hline & Median (IR) & $4.7(2.2-7.0)$ & $3.0(1.1-3.8)$ & & $2.4(1.0-3.7)$ & $1.4(0.5-2.1)$ & & $524.6(445.9-599.1)$ & $464.3(435.7-520.4)$ & \\
\hline \multicolumn{2}{|c|}{$P$ value } & 1 & 0.247 & & 0.927 & 0.247 & & 0.788 & 1 & \\
\hline
\end{tabular}




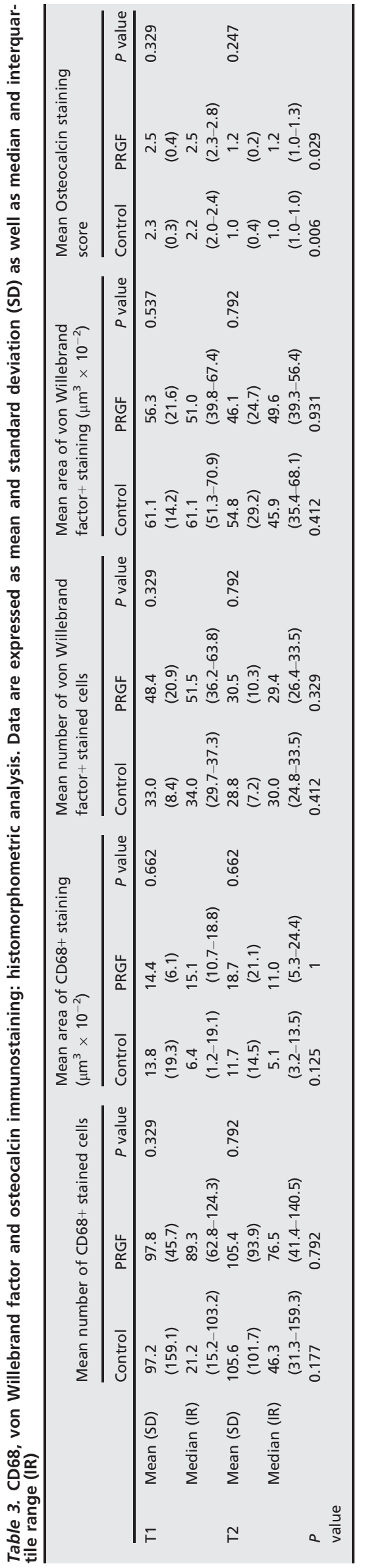

extraction sockets treated with PRGF. Twenty-eight patients lage range: 3474 years) contributing 36 extraction sockets were included in the study. Sockets were either treated with PRGF or left to spontaneous healing. Within the limits of the present study, the results indicate that patients treated with PRGF application showed (i) similar bone volume and tissue mineral content, (ii) a trend, although not statistically significant, toward a greater number of CD68+ cells (at $\mathrm{T} 1$ and $\mathrm{T} 2$ ) and vVW+ cells (at T1), and (iii) a similar OCN staining score throughout the study, compared with control patients.

In control group, spontaneous healing was characterized by a progressive increase (although not statistically significant) of BV and TMC from $\mathrm{T} 1$ to $\mathrm{T} 2$, while TMD remained similar over time. Consistently, several studies on healing sockets in humans indicated that woven bone increases during the first weeks of healing, partly replacing granulation tissue (Amler et al. 1960; Evian et al. 1982; Trombelli et al. 2008). In particular, Trombelli et al. (2008) reported that the mean proportion of the newly formed tissue occupied by bone showed an increase from $6.9 \%$ at $2-4$ weeks to $34.0 \%$ at $6-8$ weeks (Trombelli et al. 2008). The trend toward an increased bone deposition was paralleled by a significant increase in $\mathrm{OCN}$ content at $\mathrm{T} 2$ compared with $\mathrm{T} 1$. In a previous study, a higher density of OCN-positive cells lining the woven bone trabeculae was seen at samples collected at 6-8 weeks of healing compared with samples collected after 2-4 weeks (Trombelli et al. 2008). Overall, these results support an increased bone modeling activity during the first 8-10 weeks of healing, leading to the progressive deposition of woven bone from provisional matrix. In our material, new bone formation was associated with the persistence of $\mathrm{vWF}+$ cells at $\mathrm{T} 2$, which is compatible with the substantial volume still occupied by the granulation tissue observed at early healing phase (Trombelli et al. 2008). Previous findings demonstrated a similar density of vascular units (as assessed by CD31-positive cells) at 2-4 compared with 68 weeks, whereas a substantial decrease was observed only at a later stage (12-24 weeks) (Trombelli et al. 2008).

In our study protocol, PRGF was selected among the known PRP preparations for application in extraction sockets. PRGF consists of a leukocyte-free, platelet-rich preparation obtained by one-step centrifugation of plasma and treated with sodium citrate as anticoagulant, and calcium chloride as activator (Anitua et al. 2007a, 2008). Several advantages were advocated for PRGF compared with other PRP formulations (Chen et al. 2010). The use of PRGF technology obviates immunological reactions and the risk of disease transmission associated with the use of exogenous substances (e.g., thrombin). The absence of leukocytes improves the physical characteristics of the preparation and minimizes the presence of leukocyte-related proinflammatory factors (e.g., metalloproteases, acid hydrolases) (Weibrich et al. 2005; Schnabel et al. 2007; Dohan Ehrenfest et al. 2009). The use of calcium chloride as clot activator results in significantly higher concentrations of growth factors (Tsay et al. 2005).

At $\mathrm{T} 1$ and $\mathrm{T} 2$, similar BV and TMC were evident in PRGF and control groups. This observation was paralleled by the histomorphometric findings showing similar OCN scores between groups throughout the study. In contrast, recent data from human studies (Alissa et al. 2010; Rutkowski et al. 2010; Célio-Mariano et al. 2012) seem to suggest that PRP preparations, other than PRGF, may to some extent accelerate bone deposition in the early phases of the healing of extraction sockets. However, the beneficial effects on bone density tend to reduce overtime. The effect of PRP on tissue healing is a function of many variables, including platelet concentration, the volume of PRP delivered, the extent and type of injury, and the overall medical condition of the patient (Alsousou et al. 2009). The procedure for PRP preparation significantly affects the content in platelets and, consequently, growth factors levels (Weibrich et al. 2005). Previous in vitro studies have demonstrated that PRP may exert a dose-specific effect on osteoblasts and osteoclasts, cell differentiation and proliferation being affected by high platelet concentration of the preparate (Arpornmaeklong et al. 2004; Graziani et al. 2006; Cenni et al. 2010). Positive effects on in vivo bone formation were observed for platelet concentration in PRP preparations of about $10^{6}$ platelets/ $\mu$ lWeibrich et al. 2004), which exceeds those observed in PRGF preparations (Anitua et al. 2007b, 2011).

Previous observations on PRGF reported more mature bone with better organized trabeculae and greater bone regeneration in PRGF-treated extraction sockets compared with control sites at 10-16 weeks after tooth extraction (Anitua 1999). Discrepancies in the results between studies may be partly ascribed to methodological issues. In the Anitua study, healing extraction sites were not quantitatively evaluated, the analysis of re-epithelialization and bone formation/ 
maturation being merely based on histologic observations (Anitua 1999). Moreover, in five of 10 experimental sockets, PRGF treatment was combined with an additional autologous bone graft $(A B G)$. Previous studies demonstrated that ABG per se may influence the healing process in extraction sockets (Becker et al. 1994, 1996). Since in Anitua study biopsies were collected at longer time intervals compared with our study, the possibility that the effects of PRGF on new bone formation may become manifest at a later stage cannot be excluded.

Although not statistically significant, an increased density of CD68-positive cells was observed in PRGF-treated patients compared with controls at T1 and T2. Some hypotheses may be advanced to explain the macrophages/giant cells infiltrate in the presence of an autologous platelet concentrate. First, soft tissues overlying an extraction site grafted with PRGF seem to express higher levels of some pro-inflammatory cytokines (Mozzati et al. 2010), thus potentially contributing to the recruitment of monocytes/macrophages. Second, autologous platelet concentrates may favor the expression of specific adhesion molecules on the endothelial cell membrane (Cenni et al. 2009), favoring an increased chemotaxis for monocytes/macrophages. In particular, ICAM-1 recruits monocytes to the bone-healing site, so as to promote the inflammatory reaction during the first phases of bone repair. Therefore, it was suggested that PRP may contribute to the macrophage response in the inflammatory phase, which is essential for a correct bone repair (Cenni et al. 2009). In contrast, previous studies where osseous defects have been treated with a platelet-released growth factors /obtained after centrifugation of washed, thrombin-activated cells of PRP) suggest that the increased recruitment of monocytes/macrophages and osteoclastogenesis exerted by an autologous platelet concentrate may result in an impaired early bone formation (Fuerst et al. 2004).

As angiogenesis plays a pivotal role in bone healing, vWF immunostaining was used to evaluate the presence of endothelial cells and, consequently, vascular structures in the wound area. In this respect, autologous plate-

\section{References}

Alissa, R., Esposito, M., Horner, K. \& Oliver, R. (2010). The influence of platelet-rich plasma on the healing of extraction sockets: an explorative randomised clinical trial. European Journal of Oral Implantology 3: 121-134. let concentrates seem to modulate the proliferation, motility, and invasiveness of human endothelial cells (Roussy et al. 2007; Rughetti et al. 2008; Cenni et al. 2009; Mooren et al. 2010), partly through VEGF released by platelet $\alpha$-granules (Cenni et al. 2009). In particular, the proangiogenic effect has been shown in vitro for platelet concentrations similar to (Rughetti et al. 2008) or higher than (Cenni et al. 2009) those present in PRGF. Consistently, in our material, PRGF treatment resulted in a trend toward an increased amount of vWF+ cells at $\mathrm{T} 1 \mathrm{com}$ pared with control sites. These data seem to suggest that the local application of PRGF in extraction sockets may promote an angiogenic burst during the early stage of healing, which seems to subside over time.

Smokers were unevenly distributed between treatments, the prevalence being higher in PRGF group. Pre-clinical evidence indicates that smoking may affect bone metabolism at extraction sites through a modulation of the expression of genes encoding for the key molecules of bone healing. A trend toward the down-regulation of bone morphogenetic protein 2 and alkaline phosphatase and the upregulation of the receptor activator of nuclear factor kappa-B ligand/osteoprotegerin ratio was reported, suggesting that smoking could be detrimental to new bone formation (Giorgetti et al. 2010). Few clinical studies have investigated the effect of smoking on the healing of extraction sockets in humans. In this respect, Saldanha et al. (2006) showed that smoking may negatively affect radiographic bone density as well as the extent of the dimensional reduction occurring after tooth extraction. Thus, it is possible to hypothesize that PRGF may have counterbalanced the potential negative effect of smoking on early bone formation. Unfortunately, a sub-analysis to explore the role of smoking on PRGFinduced healing dynamics was not feasible owing to the limited sample size of subgroups.

In our study, the selection of all patients was based on stringent inclusion criteria; however, the treatment of all cases in PRGF group was assigned to one clinician with a documented expertize in PRGF technology. Although a selection bias owing to lack of randomization cannot be excluded, this design may have optimized the clinical efficacy of PRGF treatment as well as reduced the potential variability associated with PRGF preparation and clinical use.

In conclusion, our results indicate that patients treated with PRGF application showed (i) similar bone volume and tissue mineral content, (ii) a trend, although not statistically significant, toward a greater number of CD68+ cells (at T1 and T2) and vVW+ cells (at T1), and (iii) a similar OCN staining score throughout the study, compared with control patients. Within its limits, the present study revealed that PRGF-treated group did not show any enhancement in early (4 and 8 weeks) bone deposition compared with control group. In our study, the effect of PRGF was merely investigated in extraction sockets from single-rooted teeth or single roots of mandibular molars. Although no attempt was made to assess the volume of the extraction socket (or the extracted root) immediately after surgery, such selection may have reduced the inter-site differences in healing dynamics owing to the variability in socket dimension. On the other hand, treatment of defects of limited size may have masked and underestimated the true biologic potential of PRGF on early bone formation. Further controlled studies are needed to assess which type of post-extraction conditions, including patient characteristics as well as dimension and morphology of the socket, may represent an indication for the additional use of PRGF. Also, other potential benefits, including reduced incidence of alveolar osteitis (Rutkowski et al. 2007), lower post-extraction pain, and enhanced quality of soft tissue healing (Alissa et al. 2010), may indicate PRGF treatment in extraction sockets.

\section{Acknowledgements: This study was} supported by the Research Center for the Study of Periodontal and Peri-Implant Diseases, University of Ferrara, and a grant from Regione Emilia-Romagna (Programma Ricerca Regione - Università 2007-09). The authors have no conflict of interest to declare.

Alsousou, J., Thompson, M., Hulley, P., Noble, A. \& Willett, K. (2009). The biology of platelet-rich plasma and its application in trauma and orthopaedic surgery: a review of the literature. Journal of Bone and Joint Surgery. British volume 91: 987-996.
Amler, M.H. (1969). The time sequence of tissue regeneration in human extraction wounds. Oral Surgery Oral Medicine Oral Pathology 27: 309318. 
Amler, M.H., Johnson, P.L. \& Salman, I. (1960) Histological and histochemical investigation of human alveolar socket healing in undisturbed extraction wounds. Journal of the American Dental Association 61: 32-44.

Amler, M.H., Salman, I. \& Bungener, H. (1964). Reticular and collagen fiber characteristics in human bone healing. Oral Surgery Oral Medicine Oral Pathology 17: 785-796.

Anitua, E. (1999). Plasma rich in growth factors: preliminary results of use in the preparation of future sites for implants. International Journal of Oral and Maxillofacial Implants 14: 529-535.

Anitua, E., Sanchez, M., Merayo-Lloves, J., De la Fuente, M., Muruzabal, F. \& Orive, G. (2011). Plasma rich in growth factors (PRGF-endoret) stimulates proliferation and migration of primary keratocytes and conjunctival fibroblasts and inhibits and reverts TGF-\{beta $\} 1$-induced myodifferentiation. Investigative Ophthalmology and Visual Science 52: 6066-6073.

Anitua, E., Sánchez, M., Nurden, A.T., Zalduendo, M.M., de la Fuente, M., Azofra, J. \& Andía, I. (2007b). Platelet-released growth factors enhance the secretion of hyaluronic acid and induce hepatocyte growth factor production by synovial fibroblasts from arthritic patients. Rheumatology (Oxford) 46: 1769-1772.

Anitua, E., Sánchez, M. \& Orive, G. (2010). Potential of endogenous regenerative technology for in situ regenerative medicine. Advanced Drug Delivery Reviews 62: 741-752.

Anitua, E., Sánchez, M., Orive, G. \& Andia, I. (2008). Delivering growth factors for therapeutics. Trends in Pharmacological Sciences 29: 37-41.

Anitua, E., Sánchez, M., Orive, G. \& Andía, I. (2007a). The potential impact of the preparation rich in growth factors (PRGF) in different medical fields. Biomaterials 28: 4551-4560.

Arpornmaeklong, P., Kochel, M., Depprich, R., Kübler, N.R. \& Würzler, K.K. (2004). Influence of platelet-rich plasma (PRP) on osteogenic differentiation of rat bone marrow stromal cells. An in vitro study. International Journal of Oral and Maxillofacial Surgery 33: 60-70.

Becker, W., Becker, B.E. \& Caffesse, R. (1994). A comparison of demineralized freeze-dried bone and autologous bone to induce bone formation in human extraction sockets. Journal of Periodontology 65: 1128-1133.

Becker, W., Urist, M., Becker, B.E., Jackson, W., Parry, D.A., Bartold, M., Vincenzi, G., De Georges, D. \& Niederwanger, M. (1996). Clinical and histologic observations of sites implanted with intraoral autologous bone grafts or allografts. 15 human case reports. Journal of Periodontology 67: 1025-1033.

Boyne, P.J. (1966). Osseous repair of the postextraction alveolus in man. Oral Surgery Oral Medicine Oral Pathology 21: 805-813.

Cardaropoli, G., Araujo, M., Hayacibara, R., Sukekava, F. \& Lindhe, J. (2005). Healing of extraction sockets and surgically produced - augmented and non-augmented - defects in the alveolar ridge. An experimental study in the dog. Journal of Clinical Periodontology 32: 435-440.

Cardaropoli, G., Araujo, M. \& Lindhe, J. (2003). Dynamics of bone tissue formation in tooth extraction sites. An experimental study in dogs. Journal of Clinical Periodontology 30: 809-818.

Célio-Mariano, R., de Melo, W.M. \& CarneiroAvelino, C. (2012). Comparative radiographic evaluation of alveolar bone healing associated with autologous platelet-rich plasma after impacted mandibular third molar surgery. Journal of Oral and Maxillofacial Surgery 70: 1924.

Cenni, E., Avnet, S., Fotia, C., Salerno, M. \& Baldini, N. (2010). Platelet-rich plasma impairs osteoclast generation from human precursors of peripheral blood. Journal of Orthopaedic Research 28: 792-797.

Cenni, E., Ciapetti, G., Granchi, D., Fotia, C., Perut, F., Giunti, A. \& Baldini, N. (2009). Endothelial cells incubated with platelet-rich plasma express PDGF-B and ICAM-1 and induce bone marrow stromal cell migration. Journal of Orthopaedic Research 27: 1493-1498.

Chen, F.M., Zhang, J., Zhang, M., An, Y., Chen, F. \& Wu, Z.F. (2010). A review on endogenous regenerative technology in periodontal regenerative medicine. Biomaterials 31: 7892-7927.

Christopher, E.R. (1942). Histological study of bone healing in relation to the extraction of teeth. Northwestern University Bulletin 45: 5.

Clafin, R.S. (1936a). Healing of disturbed and undisturbed extraction wounds. Journal of American Dental Association 23: 945-959.

Dohan Ehrenfest, D.M., Rasmusson, L. \& Albrektsson, T. (2009). Classification of platelet concentrates: from pure platelet-rich plasma (P-PRP) to leucocyte- and platelet-rich fibrin (L-PRF). Trends in Biotechnology 27: 158-167.

Evian, C.I., Rosenberg, E.S., Coslet, J.G. \& Corn, H. (1982). The osteogenic activity of bone removed from healing extraction sockets in humans. Journal of Periodontology 53: 81-85.

Farina, R. \& Trombelli, L. (2012) Wound healing of extraction sockets. In: Larjava, $\mathrm{H}$. ed. Oral Wound Healing: Cell Biology and Clinical Management, 1st edition, 195-223. Hoboken, NJ: Blackwell-Wiley.

Fuerst, G., Reinhard, G., Tangl, S., Mittlböck, M., Sanroman, F. \& Watzek, G. (2004). Effect of platelet-released growth factors and collagen type I on osseous regeneration of mandibular defects. A pilot study in minipigs. Journal of Clinical Periodontology 31: 784-790.

Giorgetti, A.P., César Neto, J.B., Ruiz, K.G., Casati, M.Z., Sallum, E.A. \& Nociti, F.H., Jr. (2010). Cigarette smoke inhalation modulates gene expression in sites of bone healing: a study in rats. Oral Surgery Oral Medicine Oral Pathology Oral Radiology and Endodontics 110: 447-452.

Graziani, F., Ivanovski, S., Cei, S., Ducci, F., Tonetti, M. \& Gabriele, M. (2006). The in vitro effect of different PRP concentrations on osteoblasts and fibroblasts. Clinical Oral Implants Research 17: 212-219.

Kaigler, D., Pagni, G., Park, C.H., Tarle, S.A., Bartel, R.L. \& Giannobile, W.V. (2010). Angiogenic and osteogenic potential of bone repair cells for craniofacial regeneration. Tissue Engineering Part A 16: 2809-2820.

Kanayama, M., Kuboki, T., Akiyama, K., Nawachi, K., Miyauchi, F.M., Yatani, H., Kubota, S., Nak- anishi, T. \& Takigawa, M. (2003). Connective tissue growth factor expressed in rat alveolar bone regeneration sites after tooth extraction. Archives of Oral Biology 48: 723-730.

Kuboki, Y., Hashimoto, F. \& Ishibashi, K. (1988). Time-dependent changes of collagen crosslinks in the socket after tooth extraction in rabbits. Journal of Dental Research 67: 944-948.

Lacci, K.M. \& Dardik, A. (2010). Platelet-rich plasma: support for its use in wound healing. Yale Journal of Biology and Medicine 83: 1-9.

Lekic, P., Rojas, J., Birek, C., Tenenbaum, H. \& McCulloch, C.A. (2001). Phenotypic comparison of periodontal ligament cells in vivo and in vitro. Journal of Periodontal Research 36: 71-79.

Lin, W.L., McCulloch, C.A. \& Cho, M.I. (1994). Differentiation of periodontal ligament fibroblasts into osteoblasts during socket healing after tooth extraction in the rat. Anatatomical Records 1240: 492-506.

Lin, Z., Rios, H.F., Volk, S.L., Sugai, J.V., Jin, Q. \& Giannobile, W.V. (2011). Gene expression dynamics during bone healing and osseointegration. Journal of Periodontology 82: 1007-1017.

Mangos, J.F. (1941). The healing of extraction wounds. An experimental study based on microscopic and radiographic investigations. New Zealand Dental Journal 37: 4-24.

Mooren, R.E., Hendriks, E.J., van den eucken, J.J., Merkx, M.A., Meijer, G.J., Jansen, J.A. \& Stoelinga, P.J. (2010). The effect of platelet-rich plasma in vitro on primary cells: rat osteoblast-like cells and human endothelial cells. Tissue Engineering. Part A. 16: 3159-3172.

Mozzati, M., Martinasso, G., Pol, R., Polastri, C., Cristiano, A., Muzio, G. \& Canuto, R. (2010). The impact of plasma rich in growth factors on clinical and biological factors involved in healing processes after third molar extraction. Journal of Biomedical Materials Research. Part A 95: 741-746.

Nazaroglou, I., Stavrianos, C., Kafas, P., Matoulas, E., Upile, T., Barlas, I. \& Jerjes, W. (2009). Radiographic evaluation of bone regeneration after the application of plasma rich in growth factors in a lower third molar socket: a case report. Cases Journal 3: 9134.

Nor, J.E., Peters, M.C., Christensen, J.B., Sutorik, M.M., Linn, S., Khan, M.K., Addison, C.L., Mooney, D.J. \& Polverini, P.J. (2001). Engineering and characterization of functional human microvessels in immunodeficient mice. Laboratory Investigation: A Journal of Technical Methods and Pathology 81: 453-463.

Okuda, K., Kawase, T., Momose, M., Murata, M., Saito, Y., Suzuki, H., Wolff, L.F. \& Yoshie, H. (2003). Platelet-rich plasma contains high levels of platelet-derived growth factor and transforming growth factor-beta and modulates the proliferation of periodontally related cells in vitro. Journal of Periodontology 74: 849-857.

Park, C.H., Abramson, Z.R., Taba, M., Jr, Jin, Q., Chang, J., Kreider, J.M., Goldstein, S.A. \& Giannobile, W.V. (2007). Three-dimensional microcomputed tomographic imaging of alveolar bone in experimental bone loss or repair. Journal of Periodontology 78: 273-281.

Roussy, Y., Bertrand Duchesne, M.-P. \& Gagnon, G. (2007). Activation of human platelet-rich 
plasmas: effect on growth factors release, cell division and in vivo bone formation. Clinical Oral Implants Research 18: 639-648.

Rughetti, A., Giusti, I., D'Ascenzo, S., Leocata, P., Carta, G., Pavan, A., Dell'Orso, L. \& Dolo, V. (2008). Platelet gel-released supernatant modulates the angiogenic capability of human endothelial cells. Blood Transfusion 6: 12-17.

Rutkowski, J.L., Fennell, J.W., Kern, J.C., Madison, D.E. \& Johnson, D.A. (2007). Inhibition of alveolar osteitis in mandibular tooth extraction sites using platelet-rich plasma. Journal of Oral Implantology 33: 116-121.

Rutkowski, J.L., Johnson, D.A., Radio, N.M. \& Fennell, J.W. (2010). Platelet rich plasma to facilitate wound healing following tooth extraction. Journal of Oral Implantology 36: 11-23.

Saldanha, J.B., Casati, M.Z., Neto, F.H., Sallum, E.A. \& Nociti, F.H., Jr. (2006). Smoking may affect the alveolar process dimensions and radiographic bone density in maxillary extraction sites: a prospective study in humans. Journal of Oral and Maxillofacial Surgery 64: 1359-1365.

Sato, H. \& Takeda, Y. (2007). Proliferative activity, apoptosis, and histogenesis in the early stages of rat tooth extraction wound healing. Cells, Tissues, Organs 186: 104-111.

Schnabel, L.V., Mohammed, H.O., Miller, B.J., McDermott, W.G., Jacobson, M.S., Santangelo,
K.S. \& Fortler, L.A. (2007). Platelet-rich plasma (PRP) enhances anabolic gene expression patterns in flexor digitorum superficialis tendons. Journal of Orthopaedic Research 25: 230-240.

Trombelli, L. \& Farina, R. (2008). Clinical outcomes with bioactive agents alone or in combination with grafting or guided tissue regeneration. Journal of Clinical Periodontology 35(Suppl. 8): 117-135.

Trombelli, L., Farina, R., Marzola, A., Bozzi, L., Liljenberg, B. \& Lindhe, J. (2008). Modeling and remodeling of human extraction sockets. Journal of Clinical Periodontology 35: 630-639.

Tsay, R.C., Vo, J., Burke, A., Eisig, S.B., Lu, H.H. \& Landesberg, R. (2005). Differential growth factor retention by platelet-rich plasma composites. Journal of Oral and Maxillofacial Surgery 63: 521 -528 .

Vignoletti, F., Discepoli, N., Müller, A., de Sanctis, M., Muñoz, F. \& Sanz, M. (2012). Bone modelling at fresh extraction sockets: immediate implant placement versus spontaneous healing: an experimental study in the beagle dog. Journal of Clinical Periodontology 39: 9197.

Weibrich, G., Hansen, T., Kleis, W., Buch, R. \& Hitzler, W.E. (2004). Effect of platelet concentration in platelet-rich plasma on peri-implant bone regeneration. Bone 34: 665-671.
Weibrich, G., Kleis, W.K., Hitzler, W.E. \& Hafner, G. (2005). Comparison of the platelet concentrate collection system with the plasma-richin-growth-factors kit to produce platelet-rich plasma: a technical report. International Journal of Oral and Maxillofacial Implants 20: 118123.

\section{Supporting Information}

Additional Supporting Information may be found in the online version of this article:

Table S1. Checklist of items to include when reporting a randomized trial $(56-58)$

Please note: Wiley-Blackwell are not responsible for the content or functionality of any supporting materials supplied by the authors. Any queries (other than missing material) should be directed to the corresponding author for the article. 